\title{
Criteria for sharksfin and deltoid singularities from the plane into the plane and their applications
}

\author{
Yutaro Kabata Kentaro Saji
}

January 21, 2021

『』

\begin{abstract}
We give criteria for sharksfin and deltoid singularities from the plane into the plane. We also give geometric meanings for the conditions in the criterion of a sharksfin. As applications, we investigate such singularities appearing on an orthogonal projection of a Whitney umbrella, and a sharksfin appearing on planar motions with 2-degrees of freedom.
\end{abstract}

\section{Introduction}

The singularities of maps from the plane into the plane have been one of the fundamental subjects in the singularity theory of the smooth maps. Classification of singularities of maps from the plane into the plane has been investigated by many researchers, and useful recognition criteria for main corank one singularities are given (see for example 1, 7, 10, 12, 15 17, 25]). However, as long as the authors know, useful recognition criteria for corank two maps have not been given in the literature. In this paper, we give useful recognition criteria for "sharksfin" and "deltoid" singularities, which are the most generic singularities of corank two maps from the plane into the plane:

Theorem A. Let $f:\left(\boldsymbol{R}^{2}, 0\right) \rightarrow\left(\boldsymbol{R}^{2}, 0\right)$ be a map-germ satisfying rank $d f_{0}=0$, and let $\lambda$ be an identifier of singularities of $f$. Let $\lambda$ have a non-degenerate critical point at 0 , and let $\eta_{1}, \eta_{2}$ be vector fields which satisfy that $\eta_{1}(0)$ and $\eta_{2}(0)$ are linearly independent solutions of the Hesse quadric of $\lambda$ at 0 . Then $f$ is a sharksfin (respectively, deltoid) at 0 if and only if $\operatorname{det} \operatorname{Hess}(\lambda)(0)<0$ (respectively, $\operatorname{det} \operatorname{Hess}(\lambda)(0)>0)$,

$$
\operatorname{det}\left(\eta_{1}^{2} f, \eta_{1}^{3} f\right)(0) \neq 0 \quad \text { and } \quad \operatorname{det}\left(\eta_{2}^{2} f, \eta_{2}^{3} f\right)(0) \neq 0 .
$$

Here, $\eta f$ stands for the directional derivative of $f$ by the vector field $\eta$, and $\eta^{i} f=$ $\eta\left(\eta^{i-1} f\right)$. See Section 2.1 for the definitions of identifier of singularities and solutions of the Hesse quadric of a function at a non-degenerate critical point. We also give a

Keywords and Phrases: criteria, sharksfin, deltoid, Whitney umbrella, planar motions

Partly supported by the Japan Society for the Promotion of Science KAKENHI Grants numbered 18K03301, 20K14312 and the Japan-Brazil bilateral project JPJSBP1 20190103.

Mathematics Subject Classification 2020 Primary 57R45; Secondary 58K05 
geometric meaning of the condition (1.1) as follows: If an identifier of singularities has an index 1 non-degenerate critical point at 0 , then the singular set $S(f)$ consists of two transversal regular curves, say $\gamma_{i}(i=1,2)$. The condition (1.1) is then equivalent to both $f \circ \gamma_{i}(i=1,2)$ having $3 / 2$-cusps at 0 (Proposition 3.1).

As an application of the criteria, we investigate the geometry of an orthogonal projection of a Whitney umbrella (Section 3.4) and singularities of planar motions with 2-degrees of freedom (Section 3.5). Since maps from the plane into the plane appear in several geometric situations (see for example [6, 10, 14, 17, 24]), the authors believe these criteria will work well if one wishes to find concrete conditions for a given map to be a sharksfin or a deltoid.

See [18] for the criteria for $D_{4}^{ \pm}$-singularities of fronts, which are similar singularities of a sharksfin and a deltoid.

\section{Preliminaries and proof of criteria}

\subsection{Preliminaries}

A map-germ $f:\left(\boldsymbol{R}^{2}, 0\right) \rightarrow\left(\boldsymbol{R}^{2}, 0\right)$ is called a sharksfin (respectively, deltoid) if it is $\mathcal{A}$-equivalent to the map-germ

$$
(u, v) \mapsto\left(u v, u^{2}+v^{2}+u^{3}\right) \quad\left(\text { respectively, }\left(u v,-u^{2}+v^{2}+u^{3}\right)\right)
$$

at the origin 0 . Here two map-germs $f_{i}:\left(\boldsymbol{R}^{2}, 0\right) \rightarrow\left(\boldsymbol{R}^{2}, 0\right)(i=1,2)$ are said to be $\mathcal{A}$-equivalent if there exist a diffeomorphism-germ $\varphi$ on the source space of $f_{1}$ and a diffeomorphism-germ $\Phi$ on the target space of $f_{2}$ such that $\Phi \circ f_{1}=f_{2} \circ \varphi$ holds. We note that the map-germ $(u, v) \mapsto\left(u v, u^{2}+v^{2}+u^{3}\right)$ is $\mathcal{A}$-equivalent to $(u, v) \mapsto\left(u^{2}+v^{3}, v^{2}+u^{3}\right)$, and some of the literature uses this form for a sharksfin. A fundamental classification of map-germs from the plane into the plane is given in [15,25]. In [16], it is shown that a sharksfin and a deltoid are the only singularities of rank zero and codimension equal to or less than four. See [10, 17, 20,25] for recognition criteria for fundamental rank one singularities.

Let $f:\left(\boldsymbol{R}^{2}, 0\right) \rightarrow\left(\boldsymbol{R}^{2}, 0\right)$ be a map-germ and let rank $d f_{0}=0$. Let $\lambda$ be a non-zero functional multiple of the Jacobian of $f$. Since the zeros of $\lambda$ are the set of singular points of $f$, we call the function $\lambda$ an identifier of singularities. By the assumption rank $d f_{0}=0$, the function $\lambda$ has a critical point at 0 .

To state the criteria, we define vectors at a non-degenerate critical point of a function. Let $h:\left(\boldsymbol{R}^{2}, 0\right) \rightarrow(\boldsymbol{R}, 0)$ be a function which has a non-degenerate critical point at 0 . The solution of the Hesse quadric of $h$ at 0 is a non-zero vector $(x, y)$ which satisfies

$$
\left(\left(\begin{array}{ll}
x & y
\end{array}\right) \operatorname{Hess}(h)(0)\left(\begin{array}{l}
x \\
y
\end{array}\right)=\right) h_{u u}(0) x^{2}+2 h_{u v}(0) x y+h_{v v}(0) y^{2}=0
$$

where $(\cdot)_{u}=\partial(\cdot) / \partial u,(\cdot)_{v}=\partial(\cdot) / \partial v$ and Hess $(h)(0)$ is the Hesse matrix of $h$ at 0 . If $\operatorname{det} \operatorname{Hess}(h)(0)>0$, then there exist two $\boldsymbol{C}$-linearly independent $\boldsymbol{C}$-valued vectors $v_{1}, v_{2}$. If $\operatorname{det} \operatorname{Hess}(h)(0)<0$, then there exist two $\boldsymbol{R}$-linearly independent $\boldsymbol{R}$-valued vectors $v_{1}, v_{2}$, and these are the tangent vectors of two branch curves of the zero set of $h$ at 0 . For a diffeomorphism $\varphi:\left(\boldsymbol{R}^{2}, 0\right) \rightarrow\left(\boldsymbol{R}^{2}, 0\right)$, since the Hesse matrix of $h \circ \varphi^{-1}$ 
is ${ }^{t} J_{\varphi}(0)^{-1} \operatorname{Hess}(h)(0) J_{\varphi}(0)^{-1}$, and

$$
\left(\begin{array}{ll}
x & y
\end{array}\right) \operatorname{Hess}(h)(0)\left(\begin{array}{l}
x \\
y
\end{array}\right)={ }^{t}\left(J_{\varphi}(0)\left(\begin{array}{l}
x \\
y
\end{array}\right)\right) \operatorname{Hess}\left(h \circ \varphi^{-1}\right)(0) J_{\varphi}(0)\left(\begin{array}{l}
x \\
y
\end{array}\right)
$$

the two directions defined by solutions of the Hesse quadric of $h$ at 0 do not depend on the choice of the coordinate system on the source space. Here, ${ }^{t}()$ stands for the matrix transposition, and $J_{\varphi}$ stands for the Jacobi matrix of $\varphi$. We note that if solutions of the Hesse quadric $\eta_{i}(i=1,2)$ are complex vectors, then the left-hand sides of (1.1) may be complex numbers.

\subsection{Proof of Theorem $\mathrm{A}$}

We give a proof of Theorem A. We first simplify the expression of a rank zero-germ $f:\left(\boldsymbol{R}^{2}, 0\right) \rightarrow\left(\boldsymbol{R}^{2}, 0\right)$ by coordinate changes. Keeping in mind that we will investigate geometric meanings, we restrict to using only particular coordinate changes in the next lemma.

Lemma 2.1. If rank $d f_{0}=0$ and 0 is a non-degenerate critical point of $\lambda$, then there exist a positive local coordinate system $(u, v)$ near 0 , and an orientation preserving isometry $M$ on $\left(\boldsymbol{R}^{2}, 0\right)$, namely, $M \in \mathrm{SO}(2)$, such that

$$
M \circ f(u, v)=(u v, O(2)) .
$$

Here, $O(i)$ stands for the terms whose degrees are greater than or equal to $i$, namely, $O(i)$ is an element of

$$
\left\{f \in C^{\infty}\left(\boldsymbol{R}^{2}, 0\right) \mid \sum_{a+b \leq i-1} \frac{\partial^{a+b} f}{\partial u^{a} \partial v^{b}}(0)=0\right\} .
$$

A coordinate system is said to be positive if it has the same orientation to the standard $\boldsymbol{R}^{2}$.

Proof. Firstly we show that we may assume 0 is a non-degenerate critical point with index 1 of $f_{1}$, where $f=\left(f_{1}, f_{2}\right)$. If $f_{1}=O(3)$ or $f_{2}=O(3)$, then the Jacobian of $f$ is $O(3)$. In particular, 0 will never be a non-degenerate critical point of an identifier of singularities. Thus, we may assume $\left(f_{1}\right)_{u u}(0) \neq 0$ by a suitable rotation on the target and by choosing a positive coordinate system on the source. By a rotation on the target, $f$ can be written as $f=\left(f_{1}, b_{11} u v+b_{02} v^{2}\right)+O(3)$. If $b_{11} \neq 0$, then the claim is proved. We assume $b_{11}=0$. Since $f_{2} \neq O(3)$, it holds that $b_{02} \neq 0$. Thus $f$ can be written as $f=\left(a_{20} u^{2}+2 a_{11} u v+a_{02} v^{2}, b_{02} v^{2}\right)+O(3)$. If $a_{20} a_{02}-a_{11}^{2}<0$, then the claim is proved, and if $a_{20}=0$, then $\lambda$ degenerates. So, we may assume $a_{20} a_{02}-a_{11}^{2}>0$ and $a_{20} \neq 0$. By taking the rotation by degree $\theta$ on the target, the first component of $f$ can be written as $a_{20} \cos \theta u^{2}+2 a_{11} \cos \theta u v+\left(a_{02} \cos \theta-b_{02} \sin \theta\right) v^{2}$. One can find an angle $\theta$ such that $\cos \theta\left(\cos \theta\left(a_{20} a_{02}-a_{11}^{2}\right)-\sin \theta a_{20} b_{02}\right)<0$. This shows the claim.

Thus we may assume that 0 is a non-degenerate critical point with index 1 of $f_{1}$. By the Morse lemma, there exists a positive coordinate system $(u, v)$ such that $f$ can be written as $f=( \pm u v, O(2))$. Taking a $\pi$-rotation on the target if necessary, this shows the assertion. 
By Lemma 2.1, we see $f$ is $\mathcal{A}$-equivalent to

$$
f(u, v)=\left(u v, \varepsilon \frac{u^{2}}{2}+\frac{v^{2}}{2}+\sum_{i+j=3} \frac{a_{i j} u^{i} v^{j}}{i ! j !}+O(4)\right)(\varepsilon= \pm 1) .
$$

Lemma 2.2. Let $f$ be a map-germ of the form (2.1). When $\varepsilon=1$, then $f$ is a sharksfin if and only if $\left(a_{30}-3 a_{21}+3 a_{12}-a_{03}\right)\left(a_{30}+3 a_{21}+3 a_{12}+a_{03}\right) \neq 0$. When $\varepsilon=-1$, then $f$ is deltoid if and only if $a_{30}-3 a_{12} \neq 0$ or $3 a_{21}-a_{03} \neq 0$.

Proof. Following [16, Propositions 2.1.1 and 2.2.1], we will give a proof of this lemma. By the coordinate change $u=u_{1}+a_{12} u_{1}^{2} / 2-\varepsilon a_{21} u_{1} v_{1} / 2, v=v_{1}-a_{12} u_{1} v_{1} / 2+\varepsilon a_{21} v_{1}^{2} / 2$, we see that

$$
f\left(u_{1}, v_{1}\right)=\left(u_{1} v_{1}, \frac{\varepsilon u_{1}^{2}}{2}+\frac{v_{1}^{2}}{2}+\frac{\tilde{a}_{30} u_{1}^{3}}{6}+\frac{\tilde{a}_{03} v_{1}^{3}}{6}\right)+(O(4), O(4)),
$$

where $\tilde{a}_{30}=a_{30}+3 \varepsilon a_{12}$ and $\tilde{a}_{03}=a_{03}+3 \varepsilon a_{21}$. Let $f$ be written as in (2.2) , and $\varepsilon=1$. By the coordinate change

$$
\begin{aligned}
& u_{1}=u_{2}-v_{2}+\left(\tilde{a}_{03}-2 \tilde{a}_{30}\right) u_{2}^{2} / 12+\tilde{a}_{30} u_{2} v_{2} / 4-\left(\tilde{a}_{03}+2 \tilde{a}_{30}\right) v_{2}^{2} / 12, \\
& v_{1}=u_{2}+v_{2}+\left(\tilde{a}_{30}-2 \tilde{a}_{03}\right) u_{2}^{2} / 12-\tilde{a}_{03} u_{2} v_{2} / 4-\left(2 \tilde{a}_{03}+\tilde{a}_{30}\right) v_{2}^{2} / 12
\end{aligned}
$$

and $\Phi(X, Y)=(X+Y,-X+Y) / 2$, we see

$$
\Phi \circ f\left(u_{2}, v_{2}\right)=\left(u_{2}^{2}+\frac{\left(\tilde{a}_{03}-\tilde{a}_{30}\right) v_{2}^{3}}{12}, v_{2}^{2}+\frac{\left(\tilde{a}_{03}+\tilde{a}_{30}\right) u_{2}^{3}}{12}\right)+(O(4), O(4)) .
$$

Since a sharksfin is 3-determined ([16, Proposition 2.1.1]), $f$ in the form (2.1) is a sharksfin if and only if $\left(\tilde{a}_{03}-\tilde{a}_{30}\right)\left(\tilde{a}_{03}+\tilde{a}_{30}\right) \neq 0$, namely, $\left(a_{30}-3 a_{21}+3 a_{12}-a_{03}\right)\left(a_{30}+\right.$ $\left.3 a_{21}+3 a_{12}+a_{03}\right) \neq 0$.

Next, we show the case of a deltoid. Let $f$ be written as in (2.2) and $\varepsilon=-1$. Let $p(x)$ be the polynomial

$$
p(x)=\tilde{a}_{03} x^{5}-5 \tilde{a}_{30} x^{4}-10 \tilde{a}_{03} x^{3}+10 \tilde{a}_{30} x^{2}+5 \tilde{a}_{03} x-\tilde{a}_{30},
$$

and let $x_{0}$ be one of the solutions of $p(x)=0$. We consider the coordinate change defined by

$$
\begin{array}{r}
u_{1}=\frac{1}{96}\left(96 u_{2}-96 x_{0} v_{2}+u_{2}^{2}\left(-5 \tilde{a}_{30}\left(-3+x_{0}^{2}\right)+\tilde{a}_{03} x_{0}\left(5+x_{0}^{2}\right)\right)\right. \\
+u_{2} v_{2}\left(-\tilde{a}_{03} x_{0}^{2}\left(-7+x_{0}^{2}\right)+5 \tilde{a}_{30} x_{0}\left(-3+x_{0}^{2}\right)\right) \\
\left.+v_{2}^{2}\left(\tilde{a}_{30}\left(-9+35 x_{0}^{2}\right)+x_{0}\left(\tilde{a}_{03}\left(13-7 x_{0}^{2}\right)\right)\right)\right), \\
v_{1}=\frac{1}{96}\left(96 u_{2} x_{0}+96 v_{2}-u_{2}^{2} x_{0}\left(-5 \tilde{a}_{30}\left(-3+x_{0}^{2}\right)+\tilde{a}_{03} x_{0}\left(5+x_{0}^{2}\right)\right)\right. \\
-4 u_{2} v_{2}\left(\tilde{a}_{30}\left(3-5 x_{0}^{2}\right)+\tilde{a}_{03} x_{0}\left(5+x_{0}^{2}\right)\right) \\
\left.+v_{2}^{2} x_{0}\left(\tilde{a}_{30}\left(57-35 x_{0}^{2}\right)+\tilde{a}_{03} x_{0}\left(-61+7 x_{0}^{2}\right)\right)\right),
\end{array}
$$

and we consider

$$
\Phi(X, Y)=\left(\frac{\left(1-x_{0}^{2}\right) X+2 x_{0} Y}{\left(1+x_{0}^{2}\right)^{2}},-\frac{2\left(x_{0}^{2}-1\right)\left(2 x_{0} X+\left(x_{0}^{2}-1\right) Y\right)}{\left(1+x_{0}^{2}\right)^{2}\left(x_{0}^{2}-1\right)}\right)
$$


Then $\Phi \circ f$ is

$$
\begin{aligned}
& \left(u_{2} v_{2},-u_{2}^{2}+v_{2}^{2}+\frac{\left(\tilde{a}_{03}^{2}+\tilde{a}_{30}^{2}\right)\left(1-10 x_{0}^{2}+5 x_{0}^{4}\right)}{3 \tilde{a}_{03}\left(1+x_{0}^{2}\right)^{2}} v_{2}^{3}\right) \\
& +\frac{p\left(x_{0}\right)}{96\left(1+x_{0}^{2}\right)^{2}}\left(-2 x_{0} u_{2}^{3}+3\left(x_{0}^{2}-1\right) u_{2}^{2} v_{2}+6 x_{0} u_{2} v_{2}^{2}+\left(9+7 x_{0}^{2}\right) v_{2}^{3},\right. \\
& \quad 2\left(\left(x_{0}^{2}-1\right) u_{2}^{3}+6 x_{0} u_{2}^{2} v_{2}-3\left(x_{0}^{2}-1\right) u_{2} v_{2}^{2}\right. \\
& \left.\left.\quad 2+2\left(8 \tilde{a}_{30} / \tilde{a}_{03}+7 x_{0}\right) v_{2}^{3}\right)\right)+(O(4), O(4)) .
\end{aligned}
$$

The solutions of $5 x^{4}-10 x^{2}+1=0$ are $x_{1}= \pm(1 \pm 2 / \sqrt{5})^{1 / 2}$, and we see $5 p\left(x_{1}\right)=$ $\pm 16 \tilde{a}_{03}(10 \pm 22 / \sqrt{5})^{1 / 2}$ is not zero if $\tilde{a}_{03} \neq 0$. Since a deltoid is 3-determined ([16, Proposition 2.2.1]), $f$ in the form (2.1) is a deltoid if and only if $\tilde{a}_{30} \neq 0$ or $\tilde{a}_{03} \neq 0$, namely, $a_{30}-3 a_{12} \neq 0$ or $3 a_{21}-a_{03} \neq 0$.

Lemma 2.3. Let 0 be a non-degenerate critical point of an identifier of singularities $\lambda$ of $f:\left(\boldsymbol{R}^{2}, 0\right) \rightarrow\left(\boldsymbol{R}^{2}, 0\right)$, and let $\left(\eta_{11}, \eta_{12}\right),\left(\eta_{21}, \eta_{22}\right)$ (possibly complex) be two linearly independent solutions of the Hesse quadric of $\lambda$ at 0 . Let $\eta_{i}$ be a vector field satisfying

$$
\eta_{i}(0)=\eta_{i 1} \partial_{u}+\eta_{i 2} \partial_{v} \quad(i=1,2) .
$$

Then the condition

$$
\operatorname{det}\left(\eta_{1}^{2} f, \eta_{1}^{3} f\right)(0) \neq 0 \quad \text { and } \quad \operatorname{det}\left(\eta_{2}^{2} f, \eta_{2}^{3} f\right)(0) \neq 0
$$

does not depend on the choice of $\eta_{1}, \eta_{2}$.

Proof. The condition clearly does not depend on the choice of the coordinate system on the source space, and the condition does not change by a linear coordinate transformation on the target space. As we remarked just after Lemma 2.1, we may assume $f$ is written as in (2.1). Then the identifier of singularities is $-\varepsilon u^{2}+v^{2}+O(3)$. Since the condition does not change under a constant multiplication of the solutions of the Hesse quadric $\eta_{1}$ and $\eta_{2}$ of an identifier of singularities, we may assume $\eta_{1}=(1,1), \eta_{2}=(1,-1)$ if $\varepsilon=1$, and $\eta_{1}=(1, i), \eta_{2}=(1,-i)$ if $\varepsilon=-1$, where $i=\sqrt{-1}$. By a direct calculation,

$$
\begin{aligned}
& \xi^{2} f=\left(2 \xi_{1} \xi_{2}, \varepsilon \xi_{1}^{2}+\xi_{2}^{2}\right) \\
& \xi^{3} f=\left(3\left(\xi_{2}^{2}\left(\xi_{1}\right)_{v}+\xi_{1} \xi_{2}\left(\left(\xi_{2}\right)_{v}+\left(\xi_{1}\right)_{u}\right)+\xi_{1}^{2}\left(\xi_{2}\right)_{u}\right),\right. \\
& a_{30} \xi_{1}^{3}+3 a_{21} \xi_{1}^{2} \xi_{2}+3 a_{12} \xi_{1} \xi_{2}^{2}+a_{03} \xi_{2}^{3} \\
& \left.\quad+3 \varepsilon \xi_{1}^{2}\left(\xi_{1}\right)_{u}+3 \varepsilon \xi_{1} \xi_{2}\left(\xi_{1}\right)_{v}+3 \xi_{1} \xi_{2}\left(\xi_{2}\right)_{u}+3 \xi_{2}^{2}\left(\xi_{2}\right)_{v}\right),
\end{aligned}
$$

holds at $u=v=0$, where $\xi=\left(\xi_{1}, \xi_{2}\right)$ is a vector field, and

$$
\frac{1}{2} \operatorname{det}\left(\xi^{2} f, \xi^{3} f\right)(0)=\left\{\begin{array}{l}
a_{30}+3 a_{21}+3 a_{12}+a_{03} \quad\left(\varepsilon=1, \xi(0)=\eta_{1}=(1,1)\right) \\
-a_{30}+3 a_{21}-3 a_{12}+a_{03} \quad\left(\varepsilon=1, \xi(0)=\eta_{2}=(1,-1)\right) \\
a_{30} i-3 a_{21}-3 i a_{12}+a_{03} \quad\left(\varepsilon=-1, \xi(0)=\eta_{1}=(1, i)\right) \\
-a_{30} i-3 a_{21}+3 i a_{12}+a_{03} \quad\left(\varepsilon=-1, \xi(0)=\eta_{2}=(1,-i)\right) .
\end{array}\right.
$$

The right-hand side of (2.5) depends only on the value of $\xi(0)$. This shows the assertion. 
Proof of Theorem A. The sufficiency follows by the independence of the choice of coordinate systems and vector fields. We show the necessity. We assume the assumption of Theorem A. Since the condition does not depend on the choice of coordinate systems and vector fields, we may assume $f$ is written as in (2.1). Then by (2.5) in the proof of Lemma 2.3, the condition (1.1) in Theorem $\mathrm{A}$ is equivalent to $\left(a_{30}+3 a_{21}+3 a_{12}+a_{03}\right)\left(-a_{30}+3 a_{21}-3 a_{12}+a_{03}\right) \neq 0$ when $\varepsilon=1$, and $a_{30}-3 a_{12} \neq 0$ or $3 a_{21}-a_{03} \neq 0$ when $\varepsilon=-1$. By Lemma 2.2, we have the assertion.

\section{Geometry of a sharksfin and a deltoid}

\subsection{Geometric meanings of a criterion for a sharksfin}

We here give a geometric interpretation of condition (1.1). Let $f:\left(\boldsymbol{R}^{2}, 0\right) \rightarrow\left(\boldsymbol{R}^{2}, 0\right)$ be a map-germ with rank $d f_{0}=0$, and let an identifier of singularities $\lambda$ have an index one critical point at 0 . Then the set $\lambda^{-1}(0)$ consists of images of two transversal regular curves passing through 0 . We set these curves as $\gamma_{i}:(\boldsymbol{R}, 0) \rightarrow\left(\boldsymbol{R}^{2}, 0\right)$. A curvegerm $c:(\boldsymbol{R}, 0) \rightarrow\left(\boldsymbol{R}^{2}, 0\right)$ at 0 is a $3 / 2$-cusp if it is $\mathcal{A}$-equivalent to $t \mapsto\left(t^{2}, t^{3}\right)$. It is well-known that $c:(\boldsymbol{R}, 0) \rightarrow\left(\boldsymbol{R}^{2}, 0\right)$ is a $3 / 2$-cusp if and only if $c^{\prime}(0)=0$, and $\operatorname{det}\left(c^{\prime \prime}(0), c^{\prime \prime \prime}(0)\right) \neq 0$. Let $c:(\boldsymbol{R}, 0) \rightarrow\left(\boldsymbol{R}^{2}, 0\right)$ be a curve and 0 a $3 / 2$-cusp. The cuspidal direction of $c$ at 0 is the direction defined by $c^{\prime \prime}(0)$. The cuspidal direction bisects the cusp. Then the following proposition holds.

Proposition 3.1. Under the above setting, $f$ at 0 is a sharksfin if and only if $f \circ \gamma_{i}$ $(i=1,2)$ at 0 are both 3/2-cusps.

Proof. Since the condition and the assertion do not depend on the choice of the coordinate systems, we may assume that $f$ is written as (2.1) with $\varepsilon=1$. Then we may assume that $\gamma_{1}=\gamma_{+}=\left(t, a_{+}(t)\right), \gamma_{2}=\gamma_{-}=\left(t, a_{-}(t)\right)\left(a_{+}(0)=a_{-}(0)=0\right)$. Since $\lambda\left(t, a_{ \pm}(t)\right)=0$, we have $a_{ \pm}^{\prime}(0)= \pm 1$. We set $\hat{\gamma}_{i}(t)=f \circ \gamma_{i}(t)$. Then we see $\hat{\gamma}_{ \pm}^{\prime \prime}(0)=2( \pm 1,1), \hat{\gamma}_{1}^{\prime \prime \prime}(0)=\left(3 a_{ \pm}^{\prime \prime}(0), 3 a_{ \pm}^{\prime \prime}(0) \pm a_{30}+3 a_{21} \pm 3 a_{12}+a_{03}\right)$. Thus

$$
\frac{1}{2} \operatorname{det}\left(\hat{\gamma}_{ \pm}^{\prime \prime}(0), \hat{\gamma}_{ \pm}^{\prime \prime \prime}(0)\right)=a_{30} \pm 3 a_{21}+3 a_{12} \pm a_{03}
$$

By (2.5), we have the assertion.

\subsection{An $\mathrm{SO}(2)$-normal form}

We give a simplified form of a given rank zero germ by using diffeomorphisms on the source and isometries on the target space. Since coefficients of such forms are differential geometric invariants, this is convenient for studying the differential geometry of singularities. See $[2,4,5,8,9,11,13,14,19,22,24$ s such studies, for example. Two mapgerms $f_{i}:\left(\boldsymbol{R}^{2}, 0\right) \rightarrow\left(\boldsymbol{R}^{2}, 0\right)$ are said to be $\mathcal{R}_{+} \times \mathrm{SO}(2)$-equivalent if there exist an orientation preserving diffeomorphism-germ $\varphi:\left(\boldsymbol{R}^{2}, 0\right) \rightarrow\left(\boldsymbol{R}^{2}, 0\right)$ and an orientation preserving isometry $M$ on $\left(\boldsymbol{R}^{2}, 0\right)$, namely, $M \in \mathrm{SO}(2)$, such that $M \circ f_{1}=f_{2} \circ \varphi$ holds.

Proposition 3.2. Let $f:\left(\boldsymbol{R}^{2}, 0\right) \rightarrow\left(\boldsymbol{R}^{2}, 0\right)$ be a map-germ with rank $d f_{0}=0$, and let an identifier of singularities $\lambda$ have a non-degenerate critical point at 0 . Then $f$ is 
$\mathcal{R}_{+} \times \mathrm{SO}(2)$-equivalent to the germ

$$
\begin{gathered}
\left(u v, \frac{\varepsilon_{1} a_{20} u^{2}}{2}+\frac{\varepsilon_{2} a_{20} v^{2}}{2}+\frac{a_{30} u^{3}}{6}+\frac{a_{03} v^{3}}{6}\right)+(O(4), O(4)), \\
\left(a_{20}>0,\left(\varepsilon_{1}, \varepsilon_{2}\right) \in\{(1,1),(1,-1),(-1,1)\}\right) .
\end{gathered}
$$

We show this proposition by taking $\mathcal{R}_{+} \times \mathrm{SO}(2)$-equivalent germs step by step. First we show the following lemma.

Lemma 3.3. Let $f:\left(\boldsymbol{R}^{2}, 0\right) \rightarrow\left(\boldsymbol{R}^{2}, 0\right)$ be a map-germ satisfying the condition in Proposition 3.2. Then $f$ is $\mathcal{R}_{+} \times \mathrm{SO}(2)$-equivalent to the germ

$$
\left(u v, \frac{\varepsilon_{1} a_{20} u^{2}}{2}+a_{11} u v+\frac{\varepsilon_{2} a_{20} v^{2}}{2}+O(3)\right) \quad\left(a_{20} \neq 0, \varepsilon_{1}, \varepsilon_{2} \in\{1,-1\}\right) .
$$

Proof. By Lemma 2.1, we may assume $f$ is written as $f(u, v)=\left(u v, a_{20} u^{2} / 2+a_{11} u v+\right.$ $\left.a_{02} v^{2}+O(3)\right)$. Since 0 is a non-degenerate critical point of $\lambda$, we see $a_{20} a_{02} \neq 0$. Then by setting $u=\left|a_{02} / a_{20}\right|^{1 / 4} u_{1}, v=\left|a_{20} / a_{02}\right|^{1 / 4} v_{1}$, we see the assertion.

Lemma 3.4. Let $f:\left(\boldsymbol{R}^{2}, 0\right) \rightarrow\left(\boldsymbol{R}^{2}, 0\right)$ be a map-germ satisfying the assumption of Proposition 3.2. Then $f$ is $\mathcal{R}_{+} \times \mathrm{SO}(2)$-equivalent to the germ

$$
\left(b_{11} u v, \frac{a_{20} u^{2}}{2}+\frac{a_{02} v^{2}}{2}\right)+(O(3), O(3)) \quad\left(b_{11} a_{20} a_{02} \neq 0\right) .
$$

Proof. By Lemma 3.3, we may assume $f$ is written as (3.2). If $a_{11}=0$, then no proof is needed. We assume $a_{11} \neq 0$. We set $u=u_{1}+v_{1}, v=c u_{1}+d v_{1}(c, d \in \boldsymbol{R})$, and $M$ is the rotation matrix by degree $\theta$. Then $M \circ f(u, v)$ is

$$
\begin{aligned}
& \left(\left(2 c \cos \theta-\left(a_{20}+2 a_{11} c+a_{20} c^{2}\right) \sin \theta\right) \frac{u^{2}}{2}+* u v\right. \\
& \quad+\left(2 d \cos \theta-\left(a_{20}+2 a_{11} d+a_{20} d^{2}\right) \sin \theta\right) \frac{v^{2}}{2} \\
& \left.* u^{2}+\left(\left(a_{20}+a_{20} c d+a_{11}(c+d)\right) \cos \theta+(c+d) \sin \theta\right) u v+* v^{2}\right)+(O(3), O(3)),
\end{aligned}
$$

where $*$ stands for a real number which will not be needed in later calculations. To show the lemma, we need to solve the equation

$$
\left\{\begin{array}{l}
2 c \cos \theta-\left(a_{20}+2 a_{11} c+a_{20} c^{2}\right) \sin \theta=0 \\
2 d \cos \theta-\left(a_{20}+2 a_{11} d+a_{20} d^{2}\right) \sin \theta=0 \\
\left(a_{20}+a_{20} c d+a_{11}(c+d)\right) \cos \theta+(c+d) \sin \theta=0
\end{array}\right.
$$

with respect to $c, d, \theta$. If $\sin \theta=0$, then $c=d=0$, and the third equation of (3.5) is $a_{20}=0$. Thus $\sin \theta=0$ is not a solution. We assume $\sin \theta \neq 0$. Noticing the function $\cos \theta / \sin \theta$ takes values in $\boldsymbol{R}$, we set $t=\cos \theta / \sin \theta$. Then (3.5) is equivalent to

$$
\begin{aligned}
2 c t-\left(a_{20}+2 a_{11} c+a_{20} c^{2}\right) & =0 \\
2 d t-\left(a_{20}+2 a_{11} d+a_{20} d^{2}\right) & =0 \\
\left(a_{20}+a_{20} c d+a_{11}(c+d)\right) t+c+d & =0 .
\end{aligned}
$$


Note that the equations (3.6) and (3.7) are the same. Thus the solutions $c, d$ are the two solutions of the equation

$$
-a_{20} x^{2}+2\left(-a_{11}+t\right) x-a_{20}=0
$$

with respect to $x$. We set $c, d$ are these two solutions satisfying $d-c>0$, where we will see (3.9) has distinct real roots. Hence $c+d=2\left(-a_{11}+t\right) / a_{20}$ and $c d=1$. Substituting this into (3.8), we obtain one of the solutions

$$
t=\frac{-\left(1-a_{11}^{2}+a_{20}^{2}\right)+\sqrt{\left(1-a_{11}^{2}+a_{20}^{2}\right)^{2}+4 a_{11}^{2}}}{2 a_{11}}
$$

where $\left(1-a_{11}^{2}+a_{20}^{2}\right)^{2}+4 a_{11}^{2}>0$ is obvious. Substituting this into (3.6) and (3.7), we obtain solutions $c$ and $d$. Now we see that the equation (3.9) has two distinct real roots under (3.10). However this can be easily shown from

$$
\left(-a_{11}+t\right)^{2}-a_{20}^{2}>\frac{\left(1-a_{11}^{2}+a_{20}^{2}\right)^{2}+4 a_{11}^{2}}{4 a_{11}^{2}} .
$$

Finally, since 0 is a non-degenerate critical point of $\lambda$, neither the coefficients of $u v$ in the first component nor those of $u^{2}, v^{2}$ in the second component vanish.

Proof of Proposition 3.2. By Lemma 3.4, we may assume $f$ is written as in (3.3). By the Morse lemma, there exists a coordinate change $u=a u_{1}+b v_{1}+O(2), v=c u_{1}+d v_{1}+O(2)$ such that the first component of $f$ in (3.3) written by the coordinate system $\left(u_{1}, v_{1}\right)$ is $u_{1} v_{1}$. Then we have $a c=b d=0$, and we may assume $b=c=0$, since the case $a=d=0$ is similar. If the coordinate change reverses the orientation, we compose with $(u, v) \mapsto(-u, v)$. This implies that we may assume $f$ is written as

$$
\left(u v, \frac{a_{20} u^{2}}{2}+\frac{a_{02} v^{2}}{2}+\frac{a_{30} u^{3}}{6}+\frac{a_{21} u^{2} v}{2}+\frac{a_{12} u v^{2}}{2}+\frac{a_{03} v^{3}}{6}+O(3)\right) \quad\left(a_{20} a_{02} \neq 0\right) .
$$

If the first component is $-u v$, then we take a $\pi$-rotation on the target. By the coordinate change

$$
u=u_{1}+\frac{a_{12} u_{1}^{2}}{2 a_{02}}-\frac{a_{21} u_{1} v_{1}}{2 a_{20}}, \quad v=v_{1}-\frac{a_{12} u_{1} v_{1}}{2 a_{02}}+\frac{a_{21} v_{1}^{2}}{2 a_{20}}
$$

$f$ is written as

$$
\left(u_{1} v_{1}, \frac{a_{20} u_{1}^{2}}{2}+\frac{a_{02} v_{1}^{2}}{2}+\frac{\tilde{a}_{30} u_{1}^{3}}{6}+\frac{\tilde{a}_{03} v_{1}^{3}}{6}\right)+(O(4), O(4)) .
$$

By setting $u_{1}=\left|a_{02} / a_{20}\right|^{1 / 4} u_{2}, v_{1}=\left|a_{20} / a_{02}\right|^{1 / 4} v_{2}$, and taking $(u, v) \mapsto(v,-u)$ and $\pi$-rotation on the target if $a_{20}<0$ and $a_{02}<0$, we see the assertion.

We remark that the number of coefficients of the terms whose degrees are less than or equal to 3 in the form (3.1) in Proposition 3.2 is 3 . However, coefficients in the first component remain. When considering higher degree terms, it should be remarked that the form (3.2) in Lemma 3.3 is convenient, since its first component is just $u v$. 


\subsection{Geometric meaning of coefficients of the $\mathrm{SO}(2)$-normal form}

Let $f:\left(\boldsymbol{R}^{2}, 0\right) \rightarrow\left(\boldsymbol{R}^{2}, 0\right)$ at 0 be a sharksfin. In Proposition 3.1, we showed that the images of two branch curves of $S(f)$ are both $(2,3)$-cusps. Here we give an interpretation of the coefficients $a_{20}, a_{30}, a_{03}$ in the $\mathrm{SO}(2)$-normal form (3.1) by geometries of these $(2,3)$-cusps. As in the proof of Proposition 3.1, we denote two branch curves of $S(f)$ by $\gamma_{i}(i=1,2)$ and set $\hat{\gamma}_{i}=f \circ \gamma$. By the proof of Proposition [3.1, the cuspidal directions of $\hat{\gamma}_{i}(i=1,2)$ at 0 are linearly independent. Thus the angle between two cuspidal directions of $\hat{\gamma}_{i}(i=1,2)$ is a geometric invariant of $f$. On the other hand, let $c:(\boldsymbol{R}, 0) \rightarrow\left(\boldsymbol{R}^{2}, 0\right)$ at 0 be a $3 / 2$-cusp. The cuspidal curvature of $c$ at 0 is defined by

$$
\kappa_{\text {cusp }}(c)=\frac{\operatorname{det}\left(c^{\prime \prime}(0), c^{\prime \prime \prime}(0)\right)}{\left|c^{\prime \prime}(0)\right|^{5 / 2}} .
$$

The cuspidal curvature measures the wideness of the 3/2-cusp. See [21,23] for details. Since $\hat{\gamma}_{i}(i=1,2)$ have $3 / 2$-cusps at 0 , one can compute the cuspidal curvatures. We may assume that $f$ is written as in (3.1). Then in the same notation and by the same arguments as in the proof of Proposition 3.1, we have $a_{ \pm}= \pm t+\left(a_{30}-a_{03}\right) t^{2} /\left(4 a_{20}\right)+$ $O(3)$, and

$$
\hat{\gamma}_{ \pm}^{\prime \prime}(0)=\left( \pm 2,2 a_{20}\right), \quad \hat{\gamma}_{ \pm}^{\prime \prime \prime}(0)=\left(\frac{3\left( \pm a_{30}-a_{03}\right)}{2 a_{20}}, \frac{5 a_{30} \mp a_{03}}{2}\right)
$$

Thus

$$
\kappa_{\text {cusp }}\left(\hat{\gamma}_{ \pm}\right)=\frac{2\left( \pm a_{30}+a_{03}\right)}{\left(4+4 a_{20}^{2}\right)^{5 / 4}}
$$

and the angle $\theta_{\gamma}$ between the two cuspidal directions of $\hat{\gamma}_{i}(i=1,2)$ is

$$
\cos ^{-1}\left(\frac{\left|a_{20}^{2}-1\right|}{a_{20}^{2}+1}\right) \text {. }
$$

By (3.11), (3.12) and $a_{20}>0$, together with Proposition 3.2, the cuspidal curvatures of $\hat{\gamma}_{ \pm}$and the angle between the two cuspidal directions determines the sharksfin up to three degrees, namely, $\kappa_{\text {cusp }}\left(\hat{\gamma}_{ \pm}\right)$and $\theta_{\gamma}$ determines the $\mathcal{R}_{+} \times \mathrm{SO}(2)$-class of sharksfins up to 3 -jets. It is known that $f(S(f))$ determines the $\mathcal{R}$-class of a sharksfin, since it is a critical normalization [3,26]. On the other hand, a deltoid is not a critical normalization, and we cannot find such invariants in terms of $f(S(f))=\{0\}$.

\subsection{Projection of a Whitney umbrella}

Let $f:\left(\boldsymbol{R}^{2}, 0\right) \rightarrow\left(\boldsymbol{R}^{3}, 0\right)$ be a Whitney umbrella or equivalently, a cross cap, namely, it is $\mathcal{A}$-equivalent to the germ $(u, v) \mapsto\left(u, v^{2}, u v\right)$. A line generated by $d f_{0}\left(T \boldsymbol{R}^{2}\right)$ is called the tangent line of $f$, and a plane $P$ perpendicular to the tangent line is called the normal plane. Let $\pi: \boldsymbol{R}^{3} \rightarrow P$ be the orthogonal projection. It is known that if $f$ is a Whitney umbrella, then $\pi \circ f$ is a sharksfin or a deltoid, generically. More precisely, if $f:\left(\boldsymbol{R}^{2}, 0\right) \rightarrow\left(\boldsymbol{R}^{3}, 0\right)$ is a Whitney umbrella, then there exist a coordinate system $(u, v)$ and a rotation $\Phi$ on $\boldsymbol{R}^{3}$ such that

$$
f(u, v)=\left(u, u v+\frac{c_{3} v^{3}}{6}, \sum_{i+j=2}^{3} \frac{d_{i j} u^{i} v^{j}}{i ! j !}\right)+(0, O(4), O(4)) \quad\left(c_{3}, d_{02}>0, d_{i j} \in \boldsymbol{R}\right) .
$$


See [24] or [4]. A Whitney umbrella is said to be elliptic (respectively, hyperbolic) if $d_{20}>0$ (respectively, $d_{20}<0$ ). See [2, 4, 8, 22, 24] for the geometric meanings of other coefficients. Considering the orthogonal projection with respect to the tangent line into the normal plane, a rank zero singular point appears. A saharksfin (respectively, deltoid) appears on the projection of an elliptic (respectively, hyperbolic) Whitney umbrella. We give a precise condition for this fact in terms of the coefficients $c_{3}, d_{i j}$ $(i+j=2,3)$ in (3.13), namely, geometric information of the Whitney umbrella.

Theorem 3.5. Let $f$ be a Whitney umbrella written in the form (3.13) with $d_{20} \neq 0$. Let $\pi: \boldsymbol{R}^{3} \rightarrow \boldsymbol{R}^{2}$ be the orthogonal projection $\left(X_{1}, X_{2}, X_{3}\right) \mapsto\left(X_{2}, X_{3}\right)$. Then $\pi \circ f$ at 0 is a sharksfin if and only if $d_{20}>0$ and

$$
d_{30} \tilde{d}_{02}^{3}+3 \delta d_{21} \tilde{d}_{20} \tilde{d}_{02}^{2}+\left(3 d_{12} \tilde{d}_{20}^{2}-c_{3} \tilde{d}_{20}^{4}\right) \tilde{d}_{02}+\delta\left(d_{03}-d_{11} c_{3}\right) \tilde{d}_{20}^{3} \neq 0
$$

hold for both $\delta=1$ and $\delta=-1$, where $d_{20}=\tilde{d}_{20}^{2}, d_{02}=\tilde{d}_{02}^{2}$. On the other hand, $\pi \circ f$ at 0 is a deltoid if and only if $d_{20}<0$ and

$$
d_{30} \tilde{d}_{02}^{2}-3 d_{12} \tilde{d}_{20}^{2}-c_{3} \tilde{d}_{20}^{4} \neq 0 \quad \text { or } \quad d_{03} \tilde{d}_{20}^{2}-3 d_{21} \tilde{d}_{02}^{2}-d_{11} c_{3} \tilde{d}_{20}^{2} \neq 0,
$$

where $d_{20}=-\tilde{d}_{20}^{2}, d_{02}=\tilde{d}_{02}^{2}$.

We remark that the \pm -ambiguity of $\tilde{d}_{20}^{2}, \tilde{d}_{02}^{2}$ is included by the condition (3.14) holding for both $\delta= \pm 1$.

Proof. Let $\lambda$ be an identifier of singularities of $\pi \circ f$. If $d_{20}>0$ (note that $d_{02}>0$ in $(\underline{3.13}))$ then $\lambda=-\tilde{d}_{20}^{2} u^{2}+\tilde{d}_{02}^{2} v^{2}$. In this case, we set $\eta_{1}=\eta_{+}=\left(\tilde{d}_{02}, \tilde{d}_{20}\right), \eta_{2}=\eta_{-}=$ $\left(-\tilde{d}_{02}, \tilde{d}_{20}\right)$. Then we see

$$
\begin{aligned}
\eta_{ \pm} \eta_{ \pm}(\pi \circ f)(0) & =\left(2 \tilde{d}_{20} \tilde{d}_{02}, 2 \tilde{d}_{20} \tilde{d}_{02}\left(d_{11}+\tilde{d}_{20} \tilde{d}_{02}\right)\right) \\
\eta_{ \pm} \eta_{ \pm} \eta_{ \pm}(\pi \circ f)(0) & =\left(c_{3} \tilde{d}_{20}^{3}, d_{30} \tilde{d}_{02}^{3}+3 d_{21} \tilde{d}_{20} \tilde{d}_{02}^{2}+3 d_{12} \tilde{d}_{20}^{2} \tilde{d}_{02}+d_{03} \tilde{d}_{20}^{3}\right) .
\end{aligned}
$$

By a direct calculation, we have the assertion. One can obtain the case of $d_{20}<0$, under a similar calculation by setting $\eta_{1}=\eta_{+}=\left(i \tilde{d}_{02}, \tilde{d}_{20}\right), \eta_{2}=\eta_{-}=\left(-i \tilde{d}_{02}, \tilde{d}_{20}\right)$, where $i=\sqrt{-1}$.

The $\mathrm{SO}(2)$-normal form of $\pi \circ f$ is (we allow $\left(\varepsilon_{1}, \varepsilon_{2}\right)=(-1,-1)$ in (3.1) )

$$
\left(u v, \frac{w_{3}}{2\left|w_{1}\right|}\left|\frac{w_{2}}{w_{3}}\right|^{1 / 2} u^{2}+\frac{w_{2}}{2\left|w_{1}\right|}\left|\frac{w_{3}}{w_{2}}\right|^{1 / 2} v^{2}+\frac{w_{4}}{6} u^{3}+\frac{w_{5}}{6} v^{3}\right)+(O(4), O(4)),
$$

where

$$
\begin{aligned}
& w_{1}=-2\left(\left(d_{11}-\cot \theta\right) \cos \theta+\left(-d_{11}^{2}+d_{02} d_{20}+d_{11} \cot \theta\right) \sin \theta\right) / d_{02}, \\
& w_{2}=2\left(d_{11}+\cot \theta-x_{1}\right)(\cot \theta \cos \theta+\sin \theta) / d_{02}, \\
& w_{3}=2\left(-d_{11}+\cot \theta+x_{1}\right)(\cot \theta \cos \theta+\sin \theta) / d_{02},
\end{aligned}
$$

and $\theta$ is an angle satisfying that

$$
\cot \theta=\frac{-1+d_{11}^{2}-d_{02} d_{20}+\sqrt{4 d_{11}^{2}+\left(1-d_{11}^{2}+d_{02} d_{20}\right)^{2}}}{2 d_{11}} .
$$

One can obtain the coefficients $w_{4}$ and $w_{5}$ by following the procedure of the proof of Proposition 3.2. However the formula is quite complicated, and we do not state it here. 


\subsection{Planar motions}

As an application of the criteria, we give a concrete condition that a singular point appearing on a planar motion is a sharksfin.

Let $S^{1}$ be the 1 -dimensional torus $S^{1}=\boldsymbol{R} / 2 \pi \boldsymbol{Z}$, and let $S E(2)$ be the 3-dimensional Lie group

$$
S E(2)=\boldsymbol{R}^{2} \rtimes \mathrm{SO}(2)=\left\{(a, A) \mid A=\left(\begin{array}{cc}
\cos \theta & -\sin \theta \\
\sin \theta & \cos \theta
\end{array}\right), a \in \boldsymbol{R}^{2}, \theta \in S^{1}\right\} .
$$

We take a map-germ $\alpha:\left(\boldsymbol{R}^{2}, 0\right) \rightarrow S E(2)$, which is called a planar motion-germ with 2-degrees of freedom in the context here, see [6] for detail. Take a point $\omega \in \boldsymbol{R}^{2}$, and set a map-germ $e v_{\omega}:\left(S E(2),\left(a_{0}, A_{0}\right)\right) \rightarrow \boldsymbol{R}^{2}$ by $e v_{\omega}(a, A)=A \omega+a$. Then the composition $e v_{\omega} \circ \alpha:\left(\boldsymbol{R}^{2}, 0\right) \rightarrow \boldsymbol{R}^{2}$ traces the point $w$ by the action of $\alpha(s)$, and is called a trajectory of $\omega$ by $\alpha$ :

$$
e v_{\omega} \circ \alpha(s)=A(s) \omega+a(s),
$$

where $\alpha(s)=(a(s), A(s))$. In [6], a generic classification of singularities of $e v_{\omega} \circ \alpha$ at 0 is given when $\alpha$ at 0 is a Whitney umbrella, namely, it is $\mathcal{A}$-equivalent to $(u, v) \mapsto$ $\left(u, v^{2}, u v\right)$. Here, we consider a special case of the above motion. Let $\alpha:(\boldsymbol{R}, 0) \rightarrow S E(2)$ and $\beta:(\boldsymbol{R}, 0) \rightarrow S E(2)$ be two curves. Then the composite motion of $\alpha$ and $\beta$ is defined by

$$
\nu(u, v)=\beta(v) \alpha(u):(\boldsymbol{R}, 0) \times(\boldsymbol{R}, 0) \rightarrow S E(2)
$$

where the product $\beta(v) \alpha(u)$ is that of $S E(2)$. Composite motions are planar motions with 2-degrees of freedom. In [6], a generic classification of singularities of $e v_{\omega} \circ \nu$ at 0 is given, where a sharksfin is in the classification. It is also shown that a deltoid never appears on $e v_{\omega} \circ \nu$. We give a concrete condition for $e v_{\omega} \circ \nu$ to be a sharksfin in terms of the geometry of $\omega$ and $\alpha, \beta$, by using our criterion (Theorem $\mathrm{A}$ ) when $\alpha:(\boldsymbol{R}, 0) \rightarrow$ $(S E(2),(0, E))$ and $\beta:(\boldsymbol{R}, 0) \rightarrow(S E(2),(0, E))$ have singular points at 0 . We identify $\mathrm{SO}(2)$ with $S^{1}$ and we set $\alpha(u)=\left(\left(\tilde{a}_{1}(u), \tilde{a}_{2}(u)\right), \tilde{p}(u)\right)$ and $\beta(u)=\left(\left(\tilde{b}_{1}(v), \tilde{b}_{2}(v)\right), \tilde{q}(v)\right)$, where $\left(\tilde{a}_{1}(u), \tilde{a}_{2}(u)\right),\left(\tilde{b}_{1}(v), \tilde{b}_{2}(v)\right) \in \boldsymbol{R}^{2}, \tilde{p}(u), \tilde{q}(v) \in S^{1}$. By the assumption, we can write $\alpha(u)=\left(\left(u^{2} a_{1}(u), u^{2} a_{2}(u)\right), u^{2} p(u)\right)$ and $\beta(v)=\left(\left(v^{2} b_{1}(v), v^{2} b_{2}(v)\right), v^{2} q(v)\right)$.

Theorem 3.6. Under the above notation, 0 is a corank 2 singular point of $f=e v_{\omega} \circ \nu$. Furthermore, $e v_{\omega} \circ \nu$ at 0 is a sharksfin if and only if

$$
\begin{aligned}
& \operatorname{det}\left(\begin{array}{ccc}
1 & w_{2} & -w_{1} \\
p(0) & a_{1}(0) & a_{2}(0) \\
q(0) & b_{1}(0) & b_{2}(0)
\end{array}\right) \neq 0, \\
& \operatorname{det}\left(\begin{array}{ccc}
1 & w_{2} & -w_{1} \\
p(0) & a_{1}(0) & a_{2}(0) \\
p^{\prime}(0) & a_{1}^{\prime}(0) & a_{2}^{\prime}(0)
\end{array}\right) \neq 0, \quad \operatorname{det}\left(\begin{array}{ccc}
1 & w_{2} & -w_{1} \\
q(0) & b_{1}(0) & b_{2}(0) \\
q^{\prime}(0) & b_{1}^{\prime}(0) & b_{2}^{\prime}(0)
\end{array}\right) \neq 0 .
\end{aligned}
$$

We remark that the curve $\alpha=\left(u^{2} a_{1}(u), u^{2} a_{2}(u)\right):(\boldsymbol{R}, 0) \rightarrow\left(\boldsymbol{R}^{2}, 0\right)$ is a $3 / 2$-cusp if and only if

$$
\operatorname{det}\left(\begin{array}{ll}
a_{1}(0) & a_{2}(0) \\
a_{1}^{\prime}(0) & a_{2}^{\prime}(0)
\end{array}\right) \neq 0
$$


Proof. By definition,

$$
\begin{aligned}
f= & \left(v^{2} b_{1}(v)+u^{2} a_{1}(u) \cos \left(v^{2} q(v)\right)+w_{1} \cos \left(u^{2} p(u)+v^{2} q(v)\right)\right. \\
& -u^{2} a_{2}(u) \sin \left(v^{2} q(v)\right)-w_{2} \sin \left(u^{2} p(u)+v^{2} q(v)\right), \\
& v^{2} b_{2}(v)+u^{2} a_{2}(u) \cos \left(v^{2} q(v)\right)+w_{2} \cos \left(u^{2} p(u)+v^{2} q(v)\right) \\
& \left.+u^{2} a_{1}(u) \sin \left(v^{2} q(v)\right)+w_{1} \sin \left(u^{2} p(u)+v^{2} q(v)\right)\right) .
\end{aligned}
$$

This shows the first assertion. By a direct calculation, the determinant of the Jacobi matrix of $f$ has the factor $u v$. Thus the Hesse matrix of det $J f$ at $(0,0)$ is

$$
\left(\begin{array}{cc}
0 & h_{11} \\
h_{11} & 0
\end{array}\right)
$$

where

$$
h_{11}=\operatorname{det}\left(\begin{array}{ll}
a_{1}(0) & a_{2}(0) \\
b_{1}(0) & b_{2}(0)
\end{array}\right)+w_{1} \operatorname{det}\left(\begin{array}{ll}
a_{1}(0) & p(0) \\
b_{1}(0) & q(0)
\end{array}\right)+w_{2} \operatorname{det}\left(\begin{array}{ll}
a_{2}(0) & p(0) \\
b_{2}(0) & q(0)
\end{array}\right) .
$$

Thus we see the solutions of Hesse quadrics can be taken as $\eta_{1}=\partial_{u}$ and $\eta_{2}=\partial_{v}$. Since

$$
\begin{aligned}
f_{u u}(0,0) & =2\left(a_{1}(0)-w_{2} p(0), a_{2}(0)+w_{1} p(0)\right), \\
f_{u u u}(0,0) & =6\left(a_{1}^{\prime}(0)-w_{2} p^{\prime}(0), a_{2}^{\prime}(0)+w_{1} p^{\prime}(0)\right), \\
f_{v v}(0,0) & =2\left(b_{1}(0)-w_{2} q(0), b_{2}(0)+w_{1} q(0)\right), \\
f_{v v v}(0,0) & =6\left(b_{1}^{\prime}(0)-w_{2} q^{\prime}(0), b_{2}^{\prime}(0)+w_{1} q^{\prime}(0)\right),
\end{aligned}
$$

and calculating $\operatorname{det}\left(f_{u u}(0,0), f_{\text {uuu }}(0,0)\right)$ and $\operatorname{det}\left(f_{v v}(0,0), f_{v v v}(0,0)\right)$, we have the assertion.

\section{References}

[1] J. W. Bruce, A. A. du Plessis and C. T. C. Wall, Determinacy and unipotency, Invent. Math. 88 (1987), no. 3, 521-554.

[2] J. W. Bruce and J. West, Functions on a crosscap, Math. Proc. Cambridge Philos. Soc. 123 (1998), no. 1, 19-39.

[3] A. du Plessis, T. Gaffney and L. C. Wilson, Map-germs determined by their discriminants, Stratifications, singularities and differential equations, I (Marseille, 1990; Honolulu, HI, 1990), 1-40, Travaux en Cours, 54, Hermann, Paris, 1997.

[4] T. Fukui and M. Hasegawa, Fronts of Whitney umbrella-a differential geometric approach via blowing up, J. Singul. 4 (2012), 35-67.

[5] T. Fukui, Local differential geometry of cuspidal edge and swallowtail, to appear in Osaka J. Math.

[6] C.G. Gibson, D. Marsh and Y. Xiang, Singular aspects of general planar motions with two degrees of freedom, Internat J. Robotics Research 17, 10, (1998) 10681080. 
[7] V. V. Goryunov, Singularities of projections of complete intersections J. Soviet Math. 27 (1984) 2785-2811.

[8] M. Hasegawa, A. Honda, K. Naokawa, M. Umehara and K. Yamada, Intrinsic Invariants of Cross Caps, Selecta Math. 20, 3, (2014) 769-785.

[9] A. Honda, K. Naokawa, K. Saji, M. Umehara and K. Yamada, Duality on generalized cuspidal edges preserving singular set images and first fundamental forms, arXiv:1906.02556.

[10] Y. Kabata, Recognition of plane-to-plane map-germs, Topology Appl. 202 (2016), $216-238$.

[11] L. F. Martins and K. Saji, Geometric invariants of cuspidal edges, Canad. J. Math. 68 (2016), 445-462.

[12] T. Ohmoto and F. Aicardi, Francesca First order local invariants of apparent contours, Topology 45 (2006), no. 1, 27-45.

[13] R. Oset Sinha and K. Saji, The axial curvature for corank 1 singular surfaces, preprint, arXiv:1911.08823.

[14] R. Oset Sinha and F. Tari, On the flat geometry of the cuspidal edge, Osaka J. Math. 55 (2018), no. 3, 393-421.

[15] J. H. Rieger, Families of maps from the plane to the plane, J. London Math. Soc. (2) 36 (1987), no. 2, 351-369.

[16] J. H. Rieger and M. A. S. Ruas, Classification of $\mathcal{A}$-simple germs from $k^{n}$ to $k^{2}$, Compositio Math. 79 (1991), no. 1, 99-108.

[17] K. Saji, Criteria for singularities of smooth maps from the plane into the plane and their applications, Hiroshima Math. J. 40 (2010), no. 2, 229-239.

[18] K. Saji, Criteria for $D_{4}$ singularities of wave fronts, Tohoku Math. J. (2) 63 (2011), no. $1,137-147$.

[19] K. Saji, Normal form of the swallowtail and its applications, Internat. J. Math. 29 (2018), no. 7, 1850046, 17 pp.

[20] K. Saji, M. Umehara and K. Yamada, $A_{k}$ singularities of wave fronts, Math. Proc. Camb. Philos. Soc. 146 (2009), no. 3, 731-746.

[21] K. Saji, M. Umehara, and K. Yamada, The duality between singular points and inflection points on wave fronts, Osaka J. Math. 47 (2010), no. 2, 591-607.

[22] F. Tari, On pairs of geometric foliations on a cross-cap, Tohoku Math. J. (2) 59 (2007), no. 2, 233-258.

[23] M. Umehara, Differential geometry on surfaces with singularities, in: H. Arai, T. Sunada, K. Ueno (Eds.), The World of Singularities, Nippon-Hyoron-sha Co., Ltd., 2005, pp. 50-64 (in Japanese). 
[24] J. West, The differential geometry of the cross-cap, PhD. thesis, University of Liverpool (1995)

[25] H. Whitney, On singularities of mappings of euclidean spaces. I. Mappings of the plane into the plane, Ann. of Math. (2) 62 (1955), 374-410.

[26] L. Wirthmüller, Singularities determined by their discriminant, Math. Ann. 252 (1980), no. 3, 237-245.

[27] T. Yoshida, Y. Kabata and T. Ohmoto, Bifurcation of plane-to-plane map-germs of corank 2, Q. J. Math. 66 (2015), no. 1, 369-391.

(Y. Kabata)

School of Information and Data Sciences,

Nagasaki University,

Bunkyocho 1-14, Nagasaki, 852-8131, Japan

kabata@nagasaki-u.ac.jp

(K. Saji)

Department of Mathematics,

Graduate School of Science,

Kobe University,

Rokkodai 1-1, Nada, Kobe, 657-8501, Japan

saji@math.kobe-u.ac.jp 